\section{The Structures of Sulfomycins II and III}

\section{Jun Kohno, Noriaki Kameda, MaKi Nishio*, Akio KinUmaki ${ }^{\dagger}$ and Saburo Komatsubara}

Lead Generation Research Laboratory at Toda and

${ }^{\dagger}$ Lead Optimization Research Laboratory, Tanabe Seiyaku Co., Ltd.,

2-2-50, Kawagishi, Toda-shi, Saitama 335, Japan

(Received for publication May 8, 1996)

The isolation and biological activities of sulfur-containing antibiotics sulfomycins I, II and III from the fermentation broth of Streptomyces viridochromogenes MCRL-0368 were reported by EGAWA et al. in 1969. ${ }^{1)}$ The structure of sulfomycin I was determined on the basis of chemical degradations and NMR techniques ${ }^{2,3)}$ to be a cyclic thiopeptide antibiotic represented by thiostrepton $^{4)}$, berninamycin ${ }^{3)}$ and thiopeptin ${ }^{5)}$. The structures of sulfomycins II and III, however, remained unclear. Recently, SETo and his colleagues reported a series of new thiopeptide antibiotics, promothiocins A and $\mathrm{B}^{6)}$, geninthiocin ${ }^{7}$, thiotipin ${ }^{8)}$ and promoinducin ${ }^{9)}$, as tip $A$ promoter-inducing substances. In this paper, we wish to disclose the structures of sulfomycins II and III based on the 1D and 2D NMR studies.

Purification of sulfomycins II and III from the crude extract ${ }^{1)}$ was achieved by successive column chromatographies on silica gel, reverse-phase ODS and preparative HPLC. The molecular formulae of sulfomycins II and III were determined to be $\mathrm{C}_{54} \mathrm{H}_{52} \mathrm{~N}_{16} \mathrm{O}_{15} \mathrm{~S}_{2}$ and $\mathrm{C}_{53} \mathrm{H}_{50} \mathrm{~N}_{16} \mathrm{O}_{16} \mathrm{~S}_{2}$, respectively, by HRFAB-MS or FAB-MS (sulfomycin II; $m / z$ found 1251.3130 , calcd 1251.3140 for $\mathrm{C}_{54} \mathrm{H}_{52} \mathrm{~N}_{16} \mathrm{O}_{15} \mathrm{~S}_{2} \mathrm{Na}$, sulfomycin III; $m / z$ $\left.1253(\mathrm{M}+\mathrm{Na})^{+}\right)$and NMR data. The ${ }^{1} \mathrm{H}$ and ${ }^{13} \mathrm{C}$ NMR data of sulfomycins II and III are shown in Table 1.

The ${ }^{13} \mathrm{C}$ NMR spectrum of sulfomycin II displayed 54 signals composed of $\mathrm{CH}_{3}-\mathrm{C} \times 5, \quad \mathrm{CH}_{3}-\mathrm{O} \times 1$, $-\mathrm{CH}_{2}-\times 1,>\mathrm{CH}-\times 3,-\mathrm{CH}_{2}=\times 5,-\mathrm{CH}=\times 8,: \mathrm{C}=\times 21$ and carbonyl $\mathrm{C} \times 10$. Although the DQF-COSY data revealed only the presence of three partial structures $\left(-\mathrm{CH}=\mathrm{CH}-, \mathrm{CH}_{3}-\mathrm{CH}(\mathrm{OH})-\mathrm{CH}-\mathrm{NH}-\right.$ and $\mathrm{CH}_{3}-$

Table 1. ${ }^{1} \mathrm{H}$ and ${ }^{13} \mathrm{C}$ NMR data of sulfomycins II and III. ${ }^{\mathrm{a}}$

\begin{tabular}{|c|c|c|c|c|c|c|c|c|c|}
\hline \multirow{2}{*}{ Position } & \multicolumn{2}{|c|}{${ }^{13} \mathrm{C}(\delta)$} & \multicolumn{2}{|c|}{${ }^{1} \mathrm{H}(\delta)$} & \multirow{2}{*}{ Position } & \multicolumn{2}{|c|}{${ }^{13} \mathrm{C}(\delta)$} & \multicolumn{2}{|c|}{${ }^{1} \mathrm{H}(\delta)$} \\
\hline & II & III & II & III & & II & III & II & III \\
\hline Thiazole (1) & & & & & $5-\mathrm{C}$ & 153.7 & 154.1 & & \\
\hline $2-\mathrm{C}$ & 162.5 & 162.5 & & & $5-\mathrm{CH}_{3}$ & 11.4 & 11.4 & $2.58(\mathrm{~s})$ & $2.59(\mathrm{~s})$ \\
\hline $4-\mathrm{C}$ & 149.0 & 148.9 & & & $\mathrm{CO}$ & 159.8 & 159.7 & & \\
\hline $5-\mathrm{CH}$ & 127.2 & 127.1 & $8.54(\mathrm{~s})$ & $8.53(\mathrm{~s})$ & Dehydroalar & ne $(1)$ & & & \\
\hline $\mathrm{CO}$ & 160.6 & 160.6 & & & $\mathrm{NH}$ & & & $9.19(\mathrm{~s})$ & $9.17(\mathrm{~s})$ \\
\hline Threonine & & & & & $\alpha \mathrm{C}$ & 133.6 & 133.5 & & \\
\hline $\mathrm{NH}$ & & & $8.17(\mathrm{~d}, 8.1)^{\mathrm{b}}$ & $8.14(\mathrm{~d}, 8.1)$ & $\beta \mathrm{CH}_{2}$ & 104.9 & 104.7 & $5.71(\mathrm{~s}), 6.41(\mathrm{~s})$ & $5.70(\mathrm{~s}), 6.42(\mathrm{~s})$ \\
\hline$\alpha \mathrm{CH}$ & 59.2 & 59.2 & $4.36(\mathrm{dd}, 8.3,4.6)$ & $4.32(\mathrm{dd}, 8.3,4.0)$ & $\mathrm{CO}$ & 162.6 & 162.6 & & \\
\hline$\beta \mathrm{CH}$ & 66.2 & 66.1 & $4.18(\mathrm{~m})$ & $4.17(\mathrm{~m})$ & Oxazole (3) & & & & \\
\hline$\gamma \mathrm{CH}_{3}$ & 20.1 & 20.1 & $1.10(\mathrm{~d}, 6.9)$ & $1.08(\mathrm{~d}, 6.4)$ & NH & & & $9.90(\mathrm{~s})$ & $9.88(s)$ \\
\hline $\mathrm{OH}$ & & & $5.18(\mathrm{~d}, 5.7)$ & $5.20(\mathrm{~d}, 5.7)$ & $\alpha \mathrm{C}$ & 129.5 & 129.5 & & \\
\hline $\mathrm{CO}$ & 169.1 & 169.0 & & & $\beta \mathrm{CH}_{2}$ & 112.1 & 112.1 & $5.79(\mathrm{~s}), 5.69(\mathrm{~s})$ & $5.79(\mathrm{~s}), 5.69(\mathrm{~s})$ \\
\hline Oxazole (1) & & & & & $2-C^{2}$ & 158.2 & 158.2 & & \\
\hline NH & & & $9.31(\mathrm{~s})$ & $9.37(\mathrm{~s})$ & $4-\mathrm{C}$ & 138.9 & 138.9 & & \\
\hline$\alpha \mathrm{C}$ & 121.9 & 120.9 & & & $5-\mathrm{CH}$ & 140.1 & 140.1 & $8.65(\mathrm{~s})$ & $8.65(\mathrm{~s})$ \\
\hline$\beta \mathrm{CH}$ & 134.2 & 131.6 & $6.37(\mathrm{t}, 7.5)$ & $6.35(\mathrm{t}, 6.0)$ & Pyridine & & & & \\
\hline$\gamma \mathrm{CH}_{2} / \mathrm{CH}$ & 20.8 & 57.7 & $2.18(\mathrm{~m})$ & $4.07(\mathrm{~m})$ & $2-\mathrm{C}$ & 149.1 & 149.1 & & \\
\hline$\delta \mathrm{CH}_{3}$ & 12.7 & & $1.01(\mathrm{t}, 7.5)$ & & $3-\mathrm{C}$ & 130.7 & 130.6 & & \\
\hline $\mathrm{OH}$ & & & & $4.92(t, 5.7)$ & 4-CH & 140.2 & 140.2 & $8.70(\mathrm{~d}, 8.1)$ & $8.68(\mathrm{~d}, 8.2)$ \\
\hline $2-\mathrm{C}$ & 156.7 & 156.2 & & & $5-\mathrm{CH}$ & 121.7 & 121.7 & $8.30(\mathrm{~d}, 8.1)$ & $8.30 .(\mathrm{d}, 8.2)$ \\
\hline 4-C & 128.6 & 128.7 & & & $6-C$ & 146.7 & 146.8 & & \\
\hline $5-\mathrm{C}$ & 153.4 & 153.4 & & & $\mathrm{CO}$ & 161.4 & 161.4 & & \\
\hline $5-\mathrm{CH}_{3}$ & 11.4 & 11.4 & $2.57(\mathrm{~s})$ & $2.59(\mathrm{~s})$ & Dehydroalar & ne $(2)$ & & & \\
\hline $\mathrm{CO}$ & 161.3 & 161.2 & & & NH & & & $10.43(\mathrm{~s})$ & $10.43(\mathrm{~s})$ \\
\hline Thiazole (2) & & & & & $\alpha \mathrm{C}$ & 134.0 & 134.0 & & \\
\hline NH & & & $8.39(\mathrm{~d}, 9.5)$ & $8.41(\mathrm{~d}, 9.7)$ & $\mathrm{BCH}_{2}$ & 105.6 & 105.7 & $5.96(\mathrm{~s}), 6.59(\mathrm{~s})$ & $5.96(\mathrm{~s}), 6.58(\mathrm{~s})$ \\
\hline$\alpha \mathrm{CH}$ & 77.3 & 77.3 & $6.47(\mathrm{~d}, 9.7)$ & $6.47(\mathrm{~d}, 9.5)$ & $\mathrm{CO}$ & 162.9 & 162.9 & & . \\
\hline $\mathrm{OCH}_{3}$ & 55.3 & 55.3 & $3.27(\mathrm{~s})$ & $3.28(\mathrm{~s})$ & Dehydroalar & ne $(3)$ & & & \\
\hline $2-\mathrm{C}$ & 167.5 & 167.5 & & & $\mathrm{NH}$ & & & $10.07(\mathrm{~s})$ & $9.98(\mathrm{~s})$ \\
\hline 4-C & 148.9 & 148.9 & & & $\alpha \mathrm{C}$ & 136.8 & 136.8 & & . \\
\hline $5-\mathrm{CH}$ & 126.7 & 126.7 & $8.45(\mathrm{~s})$ & $8.47(\mathrm{~s})$ & $\mathrm{BCH}_{2}$ & 111.1 & 111.2 & $5.74(\mathrm{~s}), 5.76(\mathrm{~s})$ & $5.73(\mathrm{~s}), 5.75(\mathrm{~s})$ \\
\hline $\mathrm{CO}$ & 159.1 & 159.0 & & & $\mathrm{CO}$ & 162.1 & 162.1 & & \\
\hline Oxazole (2) & & & & & Dehydroalar & ne $(4)$ & & & \\
\hline NH & & & $10.00(\mathrm{~s})$ & $9.98(s)$ & $\mathrm{NH}$ & & & $9.08(\mathrm{~s})$ & $9.08(\mathrm{~s})$ \\
\hline$\alpha \mathrm{CH}$ & 123.5 & 123.5 & & & $\alpha \mathrm{C}$ & 134.6 & 134.6 & & \\
\hline$\beta \mathrm{CH}$ & 129.3 & 129.4 & $6.52(\mathrm{q}, 7.3)$ & $6.53(\mathrm{q}, 7.1)$ & $\mathrm{BCH}_{2}$ & 104.3 & 104.2 & $5.66(\mathrm{~s}), 6.14(\mathrm{~s})$ & $5.66(\mathrm{~s}), 6.13(\mathrm{~s})$ \\
\hline$\gamma \mathrm{CH}_{3}$ & 13.6 & 13.6 & $1.78(\mathrm{~d}, 7.5)$ & $1.77(\mathrm{~d}, 7.0)$ & $\mathrm{CO}$ & 165.0 & 165.0 & & \\
\hline $2-\mathrm{C}$ & 156.8 & 156.8 & 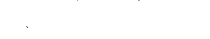 & & $\mathrm{NH}_{2}$ & & & $7.51(\mathrm{~s}), 7.94(\mathrm{~s})$ & $7.51(\mathrm{~s}), 7.93(\mathrm{~s})$ \\
\hline $4-\mathrm{C}$ & 129.2 & 129.2 & & & & & & & \\
\hline
\end{tabular}


Fig. 1. Partial structures of sulfomycin II.

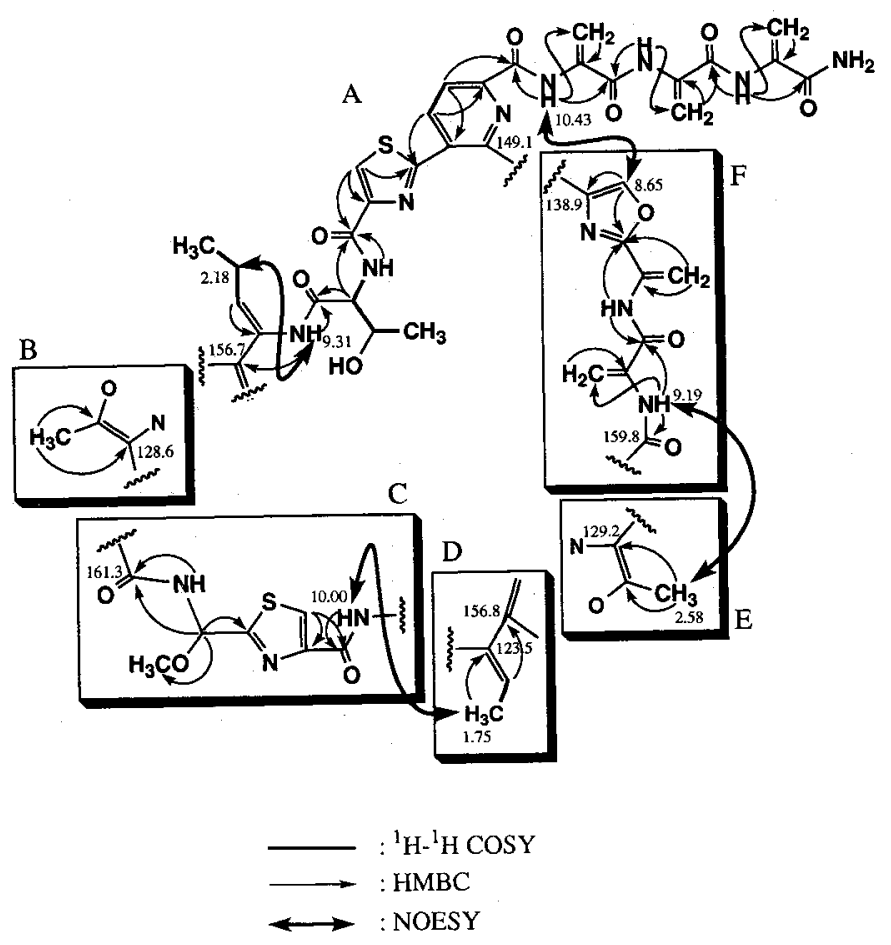

Fig. 2. Structures of sulfomycins I, II and III.

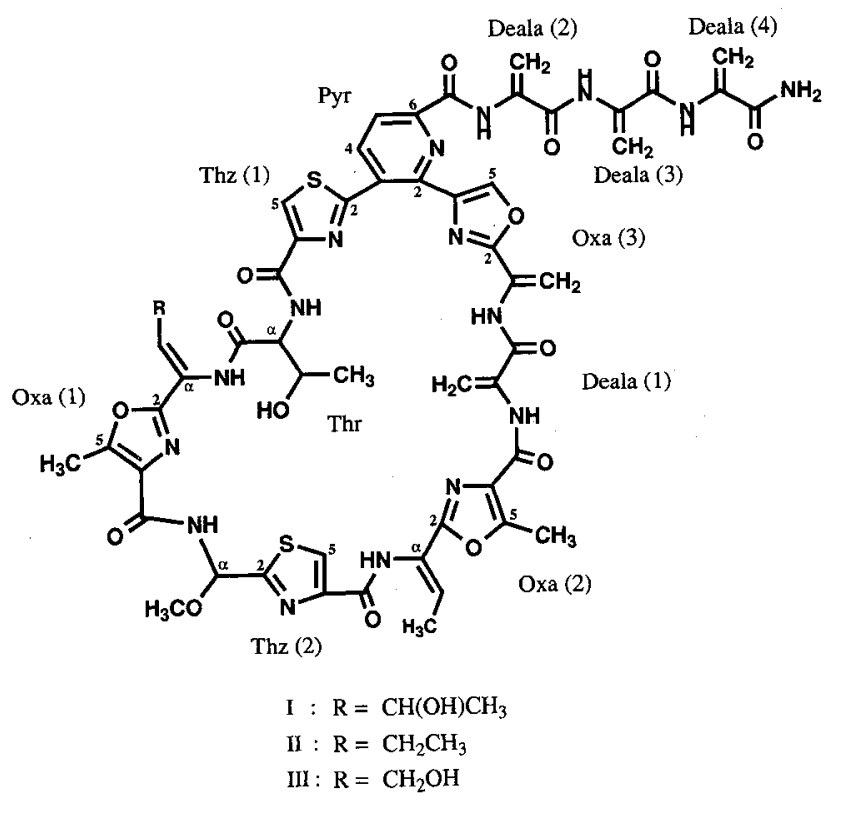

Table 2. Antimicrobial spectra of sulfomycin I, II and III and vancomycin.

\begin{tabular}{lcccc}
\hline & \multicolumn{3}{c}{$\mathrm{MIC}(\mu \mathrm{g} / \mathrm{ml})$} \\
\cline { 2 - 4 } \multicolumn{1}{c}{ Test organisms } & \multicolumn{3}{c}{ Sulfomycin } & Vancomycin \\
\cline { 2 - 4 } & \multicolumn{1}{c}{$\mathrm{I}$} & $\mathrm{II}$ & $\mathrm{III}$ & \\
\hline Staphylococcus aureus 209P JC-1 & 0.1 & 0.2 & 0.78 & 0.78 \\
Staphylococcus aureus Smith & 0.2 & 0.39 & 1.56 & 1.56 \\
MRSA TK731P & 0.05 & 0.1 & 0.39 & 0.78 \\
MRSA 252R & 0.1 & 0.2 & 1.56 & 0.78 \\
MRSA H-2-3 & 0.1 & 0.39 & 1.56 & 1.56 \\
Staphylococcus epidermidis Kawamura & 0.2 & 0.39 & 1.56 & 1.56 \\
Enterococcus faecalis ATCC29212 & 0.1 & 0.2 & 1.56 & 3.13 \\
Enterococcus faecium 173-6 & 0.05 & 0.1 & 1.56 & 1.56 \\
Escherichia coli NIHJ JC-2 & $>100$ & $>100$ & $>100$ & $>100$ \\
Klebsiella pneumoniae PCI-602 & $>100$ & $>100$ & $>100$ & $>100$ \\
Pseudomonas aeruginosa 35R & $>100$ & $>100$ & $>100$ & $>100$ \\
\hline
\end{tabular}

MIC values were determined by agar dilution method

$\left.\mathrm{CH}_{2}-\mathrm{CH}=\right),{ }^{1} \mathrm{H}^{-13} \mathrm{C}$ long range correlations observed in the HMBC spectrum exhibited the presence of six partial structures, $\mathbf{A}$ to $\mathbf{F}$, as shown in Fig. 1. The connectivities of these partial structures were established by the observation of NOEs between the olefinic proton $(\delta 8.65)$ and the amide proton $(\delta 10.43)$, the amide proton $(\delta 9.19)$ and the methyl proton $(\delta 2.58)$, and the methyl proton $(\delta 1.75)$ and the amide proton $(\delta 10.00)$.

The ${ }^{1} \mathrm{H}$ and ${ }^{13} \mathrm{C}$ NMR data along with the deduced partial structures of sulfomycin II, clearly indicated the presence of an oxazole ring in $\mathbf{B}$ and $\mathbf{E}$, respectively, in comparison with the corresponding NMR data of sulfomycin ${ }^{2)}$. The configurations of the $=\mathrm{C}=\mathrm{CH}-\mathrm{CH}_{2} \mathrm{CH}_{3}$ unit of $\mathrm{A}$ and $=\mathrm{C}=\mathrm{CH}-\mathrm{CH}_{3}$ unit of $\mathrm{D}$ were both revealed to be $Z$ by the observations of NOEs between the $\gamma$-methylene proton $(\delta 2.18)$ and the amide proton $(\delta 9.31)$, and between the $\gamma$-methyl proton $(\delta 1.75)$ and the amide proton $(\delta 10.00)$, respectively. The absolute configuration of the threonine unit was established to be $\mathrm{L}$ by analysis of the acidic hydrolysate of sulfomycin II on chiral-TLC. From these results, the structure of sulfomycin II was established as shown in Fig. 2.

The ${ }^{1} \mathrm{H}$ and ${ }^{13} \mathrm{C}$ NMR data for sulfomycin III are consistent with those of sulfomycin II except for $\gamma$ methylene and $\delta$-methyl signals of Oxa (1). In sulfomycin III, the $\delta$-methyl signal $\left(\delta_{\mathrm{C}} 12.7\right.$ and $\left.\delta_{\mathrm{H}} 1.01\right)$ in sulfomycin 
II was lacking, and an additional hydroxyl proton at $\delta 4.92$ was observed along with the downfield shift of $\gamma$-methylene signal in Oxa (1). Thus, the structure of sulfomycin III was determined as shown in Fig. 2.

The antibacterial activities of sulfomycins I, II and III and vancomycin are shown in Table 2. Sulfomycins II and III as well as sulfomycin I strongly inhibited the growth of Gram-positive bacteria including methicillinresistant Staphylococcus aureus in comparison with those of vancomycin, but are not active against Gram-negative bacteria.

\section{Acknowledgements}

We thank Dr. T. Tosa and Dr. K. Kawashima for their encouragement.

\section{References}

1) Egawa, Y.; K. Umino, Y. Tamura, M. ShimizU, K. Kaneko, M. Sakurazawa, S. Awataguchi \& T. OKuda: Sulfomycins, a series of new sulfur-containing antibiotics. I. Isolation, purification and properties. J. Antibiotics 22: $12 \sim 17,1969$

2) Abe, H.; M. Ikeda, T. Takaishi, Y. Ito \& T. OKuda: Acid hyrdolysis products of sulfomycin I. Tetrahedron
Lett. 735 736, 1977

3) Abe, H.; K Kushida, Y. Shiobara \& M. Kodama: The structures of sulfomycin $I$ and berninamycin A. Tetrahedron Lett. $1401 \sim 1404,1988$

4) Vandeputte, J. \& J. D. Dutcher: Thiostrepton, a new antibiotic. II. Isolation and chemical characterization. Antibiot. Ann. 1955/1956:560 561, 1956

5) Hensens, O. D. \& G. AlBers-SChöNBERG: Total structure of the highly modified peptide antibiotic components of thiopeptin. J. Antibiotics 36: 814 831, 1983

6) Yun, B.-S.; T. Hidaka, K. Furihata \& H. Seto: Promothiocins $\mathrm{A}$ and $\mathrm{B}$, novel thiopeptides with a tip $A$ promoter inducing activity produced by Streptomyces $\mathrm{sp}$. SF2741. J. Antibiotics 47: 510 514, 1994

7) Yun, B.-S.; T. Hidaka, K. Furihata \& H. Seto: Microbial metabolites with tip $A$ promoter inducing activity. II. Geninthiocin, a novel thiopeptide produced by Streptomyces sp. DD84. J. Antibiotics 47: 969 975, 1994

8) Yun, B.-S.; T. Hidaka, K. Furihata \& H. Sero: Thiotipin, a novel thiopeptide with a tip $A$ promoter inducing activity produced by Streptomyces sp. DT31. Tetrahedron 50: $11659 \sim 11664,1994$

9) Yun, B.-S. \& H. Seto: Promoinducin, a novel thiopeptide produced by Streptomyces sp. SF2741. Biosci. Biotech. Biochem. 59: 876 880, 1995 